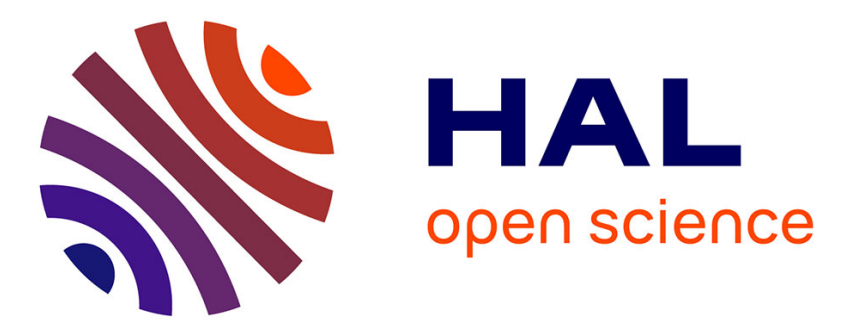

\title{
Hyperbolic-by-design self-assembled metamaterial based on block copolymers lamellar phases
}

Xuan Wang, Kevin Ehrhardt, Clemence Tallet, Marc Warenghem, Alexandre Baron, Ashod Aradian, Mortem Kildemo, Virginie Ponsinet

\section{To cite this version:}

Xuan Wang, Kevin Ehrhardt, Clemence Tallet, Marc Warenghem, Alexandre Baron, et al.. Hyperbolic-by-design self-assembled metamaterial based on block copolymers lamellar phases. Optics and Laser Technology, 2017, 88, pp.85-95. 10.1016/j.optlastec.2016.08.005 . hal-01366686v2

\section{HAL Id: hal-01366686 \\ https://hal.science/hal-01366686v2}

Submitted on 14 May 2019

HAL is a multi-disciplinary open access archive for the deposit and dissemination of scientific research documents, whether they are published or not. The documents may come from teaching and research institutions in France or abroad, or from public or private research centers.
L'archive ouverte pluridisciplinaire HAL, est destinée au dépôt et à la diffusion de documents scientifiques de niveau recherche, publiés ou non, émanant des établissements d'enseignement et de recherche français ou étrangers, des laboratoires publics ou privés. 


\title{
Hyperbolic-by-design self-assembled metamaterial based on block copolymers lamellar phases
}

\author{
Xuan WANG ${ }^{\mathrm{a}, \mathrm{b}}$, Kevin EHRHARDT ${ }^{\mathrm{a}, \mathrm{b}}$, Clémence TALLET ${ }^{\mathrm{a}, \mathrm{b}}$, Marc WARENGHEM $^{\mathrm{c}, \mathrm{d}}$, \\ Alexandre BARON ${ }^{\mathrm{a}, \mathrm{b}}$, Ashod ARADIAN ${ }^{\mathrm{a}, \mathrm{b}}$, Morten KILDEMO $^{\mathrm{e}}$, Virginie PONSINET $^{\mathrm{a}, \mathrm{b}}{ }_{*}$ \\ ${ }^{\text {a }}$ CNRS, Centre de Recherche Paul Pascal, UPR 8641, 115 Avenue Schweitzer, 33600 \\ Pessac, France \\ ${ }^{\mathrm{b}}$ Univ. Bordeaux, Centre de Recherche Paul Pascal, UPR 8641, 115 Avenue Schweitzer, \\ 33600 Pessac, France \\ ${ }^{\mathrm{c}}$ Université Lille Nord de France, 59000 Lille, France \\ ${ }^{\text {d } U n i v e r s i t e ́ ~ d ' A r t o i s, ~ U n i t e ́ ~ d e ~ C a t a l y s e ~ e t ~ d e ~ C h i m i e ~ d u ~ S o l i d e, ~ U M R ~ 8181, ~} 62307$ Lens, \\ France \\ ${ }^{\mathrm{e}}$ Physics Department, NTNU, Høgskoleringen 1, 7491 Trondheim, Norway
}

*author for correspondence: Virginie PONSINET, ponsinet@crpp-bordeaux.cnrs.fr,

Citation: Hyperbolic-by-design self-assembled metamaterial based on block copolymers lamellar phases, Xuan Wang, Kevin Ehrhardt, Clémence Tallet, Marc Warenghem, Alexandre Baron, Ashod Aradian, Morten Kildemo, Virginie Ponsinet, J. Opt. \& Laser Techn. 88, 85-95 (2017), DOI:10.1016/j.optlastec.2016.08.005

KEYWORDS: plasmonics, self-assembly, hyperbolic media, block copolymers, localized surface plasmon resonance, effective dielectric permittivity, lamellar phase

\begin{abstract}
:
Hyperbolic metamaterials use the concept of controlling the propagative modes through the engineering of the dispersion relation, and are considered highly promising to reach different metaproperties. Here we propose a novel bottom-up fabrication technique for uniaxial anisotropic metamaterials presenting a strongly anisotropic dispersion relation in the visible wavelength range, using self-assembled nanostructured block copolymers hybridized with gold nanoparticles. The materials consist in periodic lamellar stacks of period $28 \mathrm{~nm}$, of alternating layers of pure polymer (dielectric) and layers of composite of polymer loaded with a high density of $7 \mathrm{~nm}$ gold nanoparticles. The spectral variation of their anisotropic effective dielectric permittivity is determined by variableangle spectroscopic ellipsometry using appropriate effective medium models, as a function of the density of plasmonic nanoparticles. For large gold loading and close to the plasmon resonance of the nanoparticles, the lamellar stack presents ordinary and extraordinary components of the dielectric function of opposite signs. We therefore demonstrate for the first time the possibility of using a selfassembly methodology for the fabrication of bulk hyperbolic metamaterial.
\end{abstract}




\section{$\underline{\text { Introduction }}$}

The breakthroughs and innovations in the information and communication technologies rely, to a large extent, on improved performances of the devices, and their constitutive materials. Optical metamaterials lie among these envisioned breakthroughs in the near future: they are artificial materials structurally designed with the purpose of producing extraordinary optical properties. Their potential in revolutionizing the optical technologies has been largely recognized ${ }^{1,2,3}$. While precise control of both the dielectric permittivity $\varepsilon(\lambda)$ and the magnetic permeability $\mu(\lambda)$ of a 3D artificial material, in the visible or infra-red ranges, would open access to transformation optics and negative index materials, leading to the capacity of engineering the propagation of light in devices in an unprecedented manner ${ }^{2}$, these goals are still far from reach. On the other hand, by combining nanostructuration and anisotropy, it is possible to engineer novel and non-natural dispersion relations, in order to control original propagation properties. This is why a recent interest has focused on a special case of uniaxial anisotropic metamaterials, called hyperbolic materials ${ }^{4,5,6,7}$, which exhibit strongly anisotropic dispersion relations in the visible or infra-red wavelength range. These materials are considered amongst the most promising metamaterials, because of their ability to provide a multi-functional platform ${ }^{4}$ to reach different meta-properties.

For example, hyperbolic nanowire and lamellar stacked metamaterials have been studied theoretically and experimentally $y^{4,6,8,9}$. Such structures have displayed a variety of promising properties, generating a surge in the activity on the topic over the recent years: negative refraction ${ }^{10}$, super-resolution ${ }^{11}$, subwavelength modes ${ }^{12}$, perfect multi-band absorption ${ }^{13}$, optical topological transition ${ }^{14}$, epsilon-near-zero light propagation ${ }^{15}$, spontaneous emission and Purcell effect enhancement ${ }^{16,17,18,19,20}$, thermal emission engineering ${ }^{21}$ including super-Planckian regimes ${ }^{22}$, or biosensing ${ }^{23,24}$.

One very attractive property is the so-called "super-resolution" (i.e., sub-diffraction imaging), because the required structures are relatively less demanding than other metamaterials, and because superresolution could be profitable to several technological fields. Optical microscopy is indeed an essential tool in many fields such as microelectronics, biology and medicine. It is, however, hindered by the intrinsic diffraction limitation as it cannot obtain a better resolution than half the wavelength of light. This is because the finer detail-carrying, high wavevector components of the field emitted from the object are evanescent, decay exponentially in a medium with positive permittivity and permeability, and cannot contribute to the subwavelength image formation. Near-field scanning techniques ${ }^{25}$, and fluorescence-based imaging methods ${ }^{26}$ have put forward ways to circumvent this limitation and have brought optical microscopy into the nanoworld, with the fascinating and revolutionary goal to visualize the pathways of individual molecules inside living cells ${ }^{27}$. Similar intrinsic resolution limitations also hinder optical lithography, one of the most important tools equipping the semiconductor industry, ubiquitous in our societies.

In hyperbolic materials, two components of the dielectric permittivity tensor $\underline{\underline{\varepsilon}}$ have opposite signs, as if the material behaved like a metal $\left(\varepsilon_{\mathrm{i}}<0\right)$ along at least one direction and like a dielectric $\left(\varepsilon_{\mathrm{j}}>0\right)$ 
along at least another. Let us consider a uniaxial material with principal axes (x,y,z) and permittivity tensor

$$
\underline{\underline{\varepsilon}}=\left(\begin{array}{ccc}
\varepsilon_{/ /} & 0 & 0 \\
0 & \varepsilon_{/ /} & 0 \\
0 & 0 & \varepsilon_{z}
\end{array}\right) \quad \text { with } \varepsilon_{/ /}=\varepsilon_{/ /}{ }^{\prime}+\mathrm{i} \varepsilon_{/ /}{ }^{\prime} \text { and } \varepsilon_{\mathrm{z}}=\varepsilon_{\mathrm{z}}{ }^{\prime}+\mathrm{i} \varepsilon_{\mathrm{z}}{ }^{\prime} \quad \text { Equation } 1
$$

where the parallel (//) symbol denotes the (x,y)-plane. The isofrequency dispersion relation in this material is ${ }^{4,28}$

$$
\left(k_{x}{ }^{2}+k_{y}{ }^{2}-\varepsilon_{/ /} \varepsilon_{o} \mu_{o} \omega^{2}\right)\left(\left(k_{x}{ }^{2}+k_{y}{ }^{2}\right) \varepsilon_{/ /}+k_{z}{ }^{2} \varepsilon_{z}-\varepsilon_{z} \varepsilon_{/ /} \varepsilon_{o} \mu_{o} \omega^{2}\right)=0, \text { Equation } 2
$$

where the two terms describe the behavior of waves of different polarizations: polarization in the $(x, y)$ plane for the first term and polarization in a plane containing the $\mathrm{z}$ direction for the second.

The latter can be written

$$
\frac{k_{x}^{2}+k_{y}^{2}}{\varepsilon_{z}}+\frac{k_{z}^{2}}{\varepsilon_{/ /}}=k_{o}^{2}, \text { with } k_{o}^{2}=\varepsilon_{o} \mu_{o} \omega^{2}
$$

Equation 3

It exhibits a hyperboloid branch when the product $\varepsilon_{/ /}{ }^{\prime} \varepsilon_{z}{ }^{\prime}<0$, contrary to dielectrics, for which this relation defines a closed ellipsoidal shape (spherical if isotropic). This peculiar shape allows notably for the propagation of large magnitude wavevectors, carrying details finer than half the wavelength, otherwise corresponding to evanescent non-propagative waves in a usual dielectric. Hyperbolic material fabrication usually relies on metal deposition within top-down produced uniaxial nanostructures, either lamellar or cylindrical, and their permittivity tensor is usually inferred from basic effective medium models. The spectral tunability of the effective medium properties of the material is limited by the large spectral dispersion of the optical properties of the metal, which generally follows the Drude model.

One of the main bottlenecks in the research on metamaterials and hyperbolic materials in particular remains the lack of robust, simple and large scale fabrication technologies which permit tailoring final optical properties at chosen frequency ranges (including visible frequencies), and setting the technological basis for the development of devices: the field is open to innovative fabrication proposals. The powerful 'top-down' techniques such as nanolithography have been successful in manufacturing nanostructured surfaces and in evidencing meta-material properties, such as hyperlensing ${ }^{29}$ and cloaking ${ }^{1}$, at wavelengths larger or close to the visible domain ${ }^{30}$ and even at ultraviolet frequencies ${ }^{31}$. The use of chemistry and self-assembly of metallic nanoparticles, acting as plasmonic resonators, into dense ordered structures, was anticipated as a promising 'bottom-up' alternative $^{32,33}$, especially in order to produce large-scale, 3D and tunable metamaterials. The versatility of chemical synthesis and the diverse mechanisms of self-assembly can be combined to produce materials with both nanostructuration and anisotropy, presenting novel propagation properties in the visible or infrared ranges. 
In particular, diblock copolymers ${ }^{34,35}$ have long been known to self-assemble into nanomaterials with strong structural anisotropy. They are macromolecules made of two molecular chains of distinct chemical nature linked together, called the blocks, and present solid state spontaneous organizations with long-range order and tunable characteristic sizes, ranging typically from a few nanometers to a few hundred nanometers. The morphology is selected mostly in relation with the volume fractions of the blocks $f_{\mathrm{A}}$ and $f_{\mathrm{B}}=1-f_{\mathrm{A}}$, due to interfacial curvature effects ${ }^{35,36}$, among the choice of body-centered cubic array of spherical cores, bicontinuous gyroid, hexagonally packed cylinders and lamellae. It is thus largely possible to design a desired material by choosing the chemical nature and the length of the blocks in order to obtain a given morphology, a given characteristic size, as well as a given chemical functionality. Using such ordered block copolymer phases for optical applications is limited by the usually low dielectric contrast between the blocks: the refractive index of almost all easily accessible polymers is in the range $1.48-1.61$. One way of improving the contrast, among others ${ }^{37,38}$, or of conferring specific optical properties, is to disperse adequate nanoparticles within the ordered polymer phase in such a way that the particles get ordered by the matrix. This is what is reported in this work, in which we show that nanocomposites based on metal nanoparticles embedded in a dielectric host, can present a strongly anisotropic structure, as well as spectrally selective optical anisotropy, as a consequence of the spectral tunability of the plasmon resonance.

Indeed, we propose in this article a novel fabrication technique for lamellar metal-dielectric nanocomposites using self-assembled block copolymer nanostructures, and we demonstrate the relevance of such bottom-up methodology for the fabrication of hyperbolic metamaterials. We first describe the preparation and structural study of thin films of self-assembled nanocomposites of block copolymers and gold nanoparticles prepared by wet chemistry. We then present the study of the relation between the structure of the composites, and in particular the density of the gold nanoparticles, and the anisotropy of their effective dielectric permittivity tensor, which is extracted from variableangle spectroscopic ellipsometry. We finally discuss the hyperbolic nature of the self-assembled material.

\section{$\underline{\text { I. Nanostructure design and fabrication }}$}

As a first requirement, a structural uniaxial symmetry appears highly desirable, in order to obtain a hyperbolic regime of optical anisotropy. Diblock copolymer thin film engineering ${ }^{39,40}$ offers a genuine technological platform for the generation of nanostructured thin films with different morphologies of the domains. Two specific thin film morphologies are particularly attractive here, which present a high degree of order and a uniaxial symmetry: the lamellar phase in parallel alignment, which we study in the following, and the phase of hexagonally-packed cylinders in perpendicular orientation, which has been considered for applications including lithography masks ${ }^{41,42,43}$ and enhanced patterned LEDs ${ }^{44}$. A second requirement, for the light propagation properties to be described by a homogenized dispersion relation, is that the internal structure of the material presents a subwavelength characteristic size: 
$d_{o}<<\lambda$. This can be easily achieved with self-assembled block copolymer structures, with an appropriate choice of the total chain length.

We have studied several poly(styrene)-b-poly(2-vinyl pyridine) (PS-P2VP) copolymers, of different block lengths. A small-angle X-ray scattering study of thick samples of these copolymers (see Supplementary Information, Figure S1) shows the signatures of phases of either stacked lamellae or hexagonally-packed cylinders, depending on the ratio between the block lengths. The characteristic structural sizes, as given by the center-to-center distance between P2VP domains, range from 37 to 84 $\mathrm{nm}$. As expected ${ }^{35}$, a lamellar nanostructure is obtained when both blocks have similar volume fraction $f_{\mathrm{A}} \sim f_{\mathrm{B}} \sim 0.5$. Finally, we choose to work with the lamellar PS-P2VP copolymer with both blocks of molar mass $25 \mathrm{~kg} / \mathrm{mol}$, which spontaneously develops a multilayered structurally uniaxial thin film of alternate poly(styrene) and poly(2-vinyl pyridine) layers with a period of $37 \mathrm{~nm}$ in the bulk. We use a process combining spin-coating of a relatively dilute polymer solution on a selective surface (silicon wafer having a higher affinity for P2VP rather than PS) and thermal annealing, which produces a flat and homogeneous film with a parallel alignment of the organized structures, throughout the thickness of the film between 200 and $700 \mathrm{~nm}$. This ordered structure spans an area as large as the spin-coating process can produce (we use $15 \times 15 \mathrm{~mm}^{2}$ wafers).

The third requirement to obtain a hyperbolic medium, which is not provided by the simple copolymer self-assembly, is to induce dielectric permittivity of opposite sign depending on the probed direction. Thus, the combination of a metallic response $(\varepsilon<0)$ and a dielectric response $(\varepsilon>0)$ is necessary. Reported examples of multilayer hyperbolic materials have been fabricated by alternate deposition of metal and dielectric materials by electron-beam or sputter deposition in vacuum. A self-assembled block copolymer lamellar phase equivalent requires a pseudo-metallic nature for one of the layer types, which we obtain by doping one of the polymer phase with metallic nanoparticles, thus producing alternate stacks of pure polymer layers (polystyrene, referred to as PS in the following), and composite layers of gold nanoparticles and poly(2-vinyl pyridine) (referred to as NC for Nano-Composite layers) (see Figures 1 and 4).

The optical properties of nanocomposites of gold particles and polymer with no order, equivalent to the NC layers of the lamellar stacks, have been studied previously, using spectroscopic ellipsometry. We showed that such nanocomposite films present a plasmon resonance due to the metallic nanoparticles ${ }^{45}$ and that the resonance amplitude increases as the gold volume fraction in the nanocomposite increases. As the resonance deepens, the system will ultimately reach $\varepsilon<0$ in some frequency range: in fact, the Maxwell Garnett Effective Medium Approximation (MG-EMA), although not truthfully applicable for such high-fractions, suggests this can roughly occur for gold volume fractions beyond $25 \%$. In order to transform one of the block copolymer domain into such a pseudo-metallic nanocomposites, we can choose among several routes ${ }^{46,47,48}$ for selective nanoparticle incorporation. Here, we developed an impregnation methodology, adapted from the literature ${ }^{49,50}$, in which a previously ordered and aligned sturdy copolymer nanostructure can be selectively swollen 
with a precursor solution. More precisely, the film-bearing wafer is dipped into an $\mathrm{Au}$ salt $\left(\mathrm{HAuCl}_{4}\right)$ solution and then into a reducing agent $\left(\mathrm{NaBH}_{4}\right)$ solution. Due to the strong insolubility of PS in polar solvents, on the one hand, and the strong affinity of P2VP to Au, on the other hand, Au nanoparticles form selectively within the P2VP layers, thus producing a structure of alternating pure PS and (NC) (Au nanoparticles:P2VP nanocomposite) layers. One of the advantages of this infiltration procedure is that we can slowly increase, in a controlled way, the volume fraction of nanoparticles in the (NC) layers, by repeating the double dipping process $\mathrm{N}$ times. Each cycle increases the purple color of the film, related to the localized surface plasmon resonance of the nucleated gold nanoparticles. Structural and optical studies of the film were performed at different steps along the fabrication process, so that the same film was investigated for different values of $\mathrm{N}$, which we will refer to in the following as "kinetic" measurements.

Using scanning electron microscopy (SEM), we fully characterize the structure of the studied multistack films. As can be seen on an example for N=5 (Figure 1), SEM observation clearly evidenced the contrast in electron density between adjacent layers in the multilayer film, which can unambiguously be attributed to the selective gold loading of the P2VP layer. More precisely, the film is composed of 9 bilayers (PS)+(NC) of thickness $14+14=28 \mathrm{~nm}$, with an additional (NC) layer of 6 $\mathrm{nm}$ along the substrate surface and an additional (PS) layer of $6 \mathrm{~nm}$ along the air interface ${ }^{51}$. As demonstrated by the SEM images, the films are structurally uniaxial and homogeneous and we can define their dielectric permittivity tensor with the ordinary (parallel to the substrate, $\varepsilon_{\text {ord }}=\varepsilon_{/ /}$) and extraordinary (normal to the substrate, $\varepsilon_{\text {extraord }}=\varepsilon_{\mathrm{z}}$ ) components.

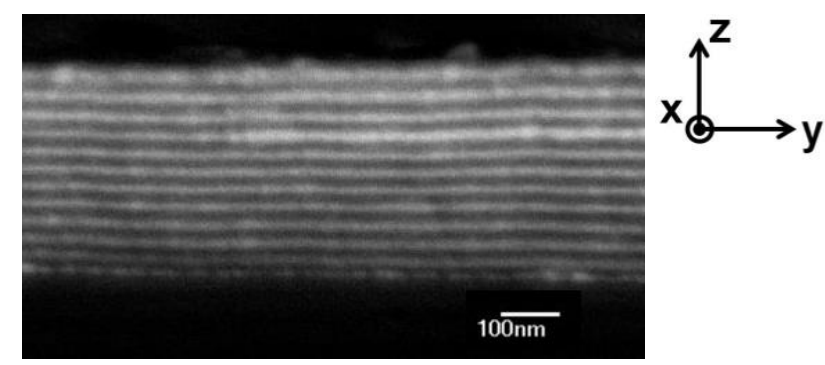

Figure 1. Backscattering scanning electron microscopy side-view image (SEM) of a $370 \mathrm{~nm}$-thick film of alternating layers of poly(styrene) (PS, appearing black) and $\mathrm{Au}$ nanoparticles: $\mathrm{P}_{2} \mathrm{VP}$ nanocomposite ( $\mathrm{NC}$, appearing white). The lower and upper black domains of the micrograph are the substrate and the air, respectively. The trihedral indicates the ordinary $((\mathrm{x}, \mathrm{y})$ or //) and extraordinary $(\mathrm{z})$ directions.

We can follow the structure of a sample as the amount of gold is increased in the film, by repeating the cycle of salt impregnation and reduction within the lamellar matrix $\mathrm{N}$ times. Figure 2 presents the evolution of the structure as followed by SEM. We can thus check that the lamellar structure is preserved and unchanged for all values of $\mathrm{N}$ for which the film was imaged. The contrast of the images cannot be quantitatively compared to confirm the increase of the gold volume fraction. It is noteworthy that the thickness of the NC layers does not show any detectable increase upon gold 
loading with increasing $\mathrm{N}$. This can be understood as due to a reorganization of the polymer chains conformation, as when such films are swollen with solvent, as well as a possible degradation of the P2VP segments involved in the chemical reduction of the gold salt. Beyond $\mathrm{N}=20$, the SEM micrographs show the evidence of an uncontrolled gold deposit on the upper surface of the film.
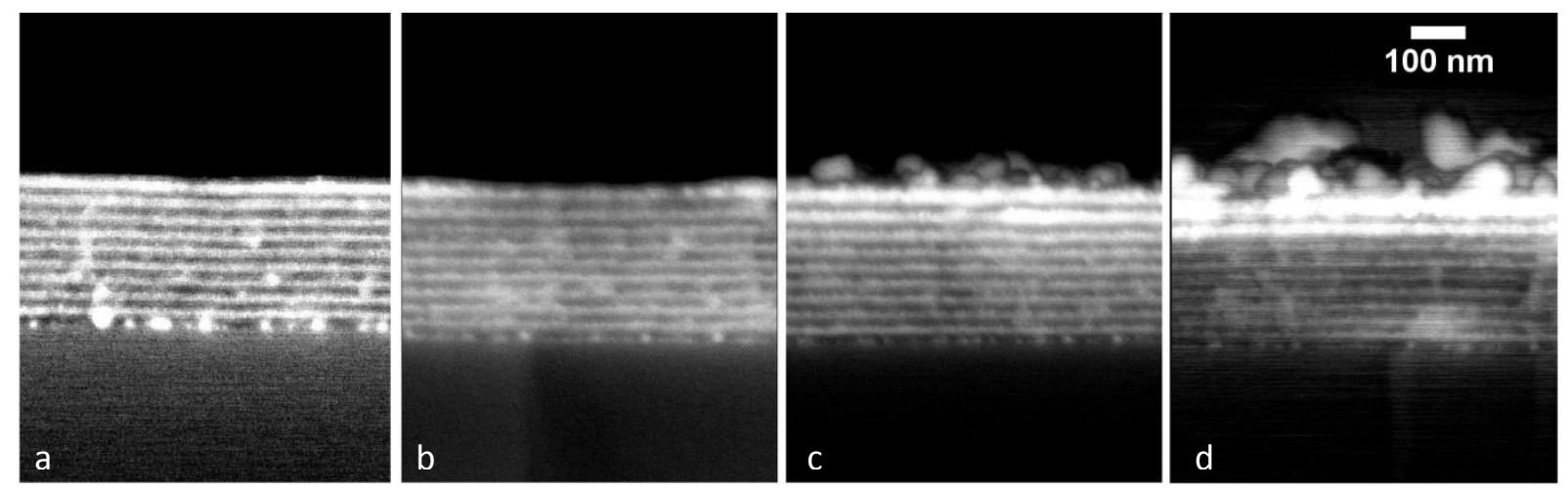

Figure 2. Backscattering scanning electron microscopy side-view image (SEM) of the $265 \mathrm{~nm}$-thick film of alternating layers of pure polymer (PS, appearing black) and of Au nanoparticles:P2VP nanocomposite (NC, appearing white), for a number of cycles of gold impregnation and reduction of $\mathrm{N}=5$ (a), 10 (b), 20 (c), 30 (d).

In order to gain more precise information on the shape and nature of the gold objects introduced within the lamellar stack, which SEM images do not provide due to lack of resolution, we performed complementary studies by transmission electron microscopy (TEM) as well as SAXS on dispersions obtained by full dissolution of the samples in a good solvent of the diblock copolymer at the end of the fabrication process. Both techniques show that the NC layers are composed of individual gold nanoparticles (NPs) of relatively homogeneous size (diameter D $\sim 7 \pm 2 \mathrm{~nm}$ ). Figure 3 shows an example of the results obtained by TEM in the case of $\mathrm{N}=25$. We can therefore infer that such $7 \mathrm{~nm}$ gold NPs are dispersed with no specific order and surrounded by a P2VP matrix, in the NC layers.

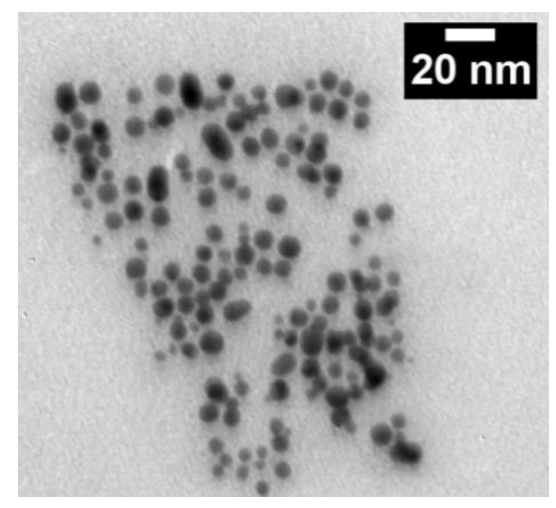

Figure 3. Transmission electron microscopy (TEM) image obtained with a grid on which was deposited a drop of the dispersion obtained by full dissolution of a film, at the step $\mathrm{N}=20$ of the fabrication process, in a good solvent of the diblock copolymer. The image evidences gold nanoparticles of mean diameter $7 \mathrm{~nm}$. Note that this observation technique being destructive, it was performed on different samples than the ones studied by SEM and VASE. 


\section{Methodology for the extraction of the optical properties}

\section{II.1. Experimental details}

We study the samples using variable angle spectroscopic ellipsometry (VASE) in reflection with a phase modulated spectroscopic ellipsometer on the spectral range [450 - $1900 \mathrm{~nm}$ ] (UVISEL from Horiba Scientific). We use the UVISEL II $\left(A=45^{\circ} ; \mathrm{M}=0\right)$ and UVISEL III $\left(\mathrm{A}=45^{\circ} ; \mathrm{M}=45^{\circ}\right)$ configurations, where $\mathrm{A}$ and $\mathrm{M}$ denote the azimuthal orientations of the input polarizer and photoelastic modulator, respectively, with respect to the plane of incidence. We acquire the ellipsometric quantities $\mathrm{I}_{\mathrm{s}}=\sin 2 \Psi \sin \Delta, \mathrm{I}_{\mathrm{c}}=\sin 2 \Psi \cos \Delta$ and $\mathrm{I}_{\mathrm{c}}{ }^{\prime}=\cos 2 \Psi$, where $\Psi$ and $\Delta$ are the two ellipsometric angles, defined by $r_{p} / r_{s}=\tan \Psi \mathrm{e}^{\mathrm{i} \Lambda}$, with $r_{p}$ and $r_{s}$ the complex reflection coefficients of the amplitude of the p-polarized (i.e., in the plane of incidence) and s-polarized (perpendicular to the plane of incidence) waves respectively. Three values of the incidence angle $\theta_{0}=55^{\circ}, 65^{\circ}$ and $75^{\circ}$ were used and analyzed simultaneously. The spot size was $1 \mathrm{~mm}$ and the measured data were checked to be similar at three different locations on the samples. It is noted that the spectroscopic ellipsometry (SE) experiment in this configuration only probes low modulus wave-vectors with $k_{x}=k_{o} \sin \theta_{o}$.

The "kinetic" VASE information was obtained by recording the full spectra every five gold loading cycles. This turns out to be very important information about the stacked structure as it is progressively evolving upon increased gold absorption in the layers. The VASE data, for one studied film, are shown on Figure 4, where the ellipsometric angles $\Psi$ and $\Delta$ are plotted for $\theta_{0}=65^{\circ}$ as a function of the photon energy between 0.6 and $2.8 \mathrm{eV}$, and for increasing values of $\mathrm{N}$ between 0 and 25. For the initial lamellar structure (before infiltration, $\mathrm{N}=0$ ), one can see periodic oscillations typical of interferences driven by reflections at the air/film and film/substrate interfaces, as both PS and P2VP polymers are transparent in the studied energy range. As $\mathrm{N}$ is increased, the extrema shift toward lower energies and the fringe spectral separation decreases indicating an evolution possibly combining an increase in the total film thickness and a change in the effective dielectric function of the NC (Au nanoparticles:P2VP) layers. Moreover, their amplitude decreases markedly as the volume fraction of $\mathrm{Au}$ increases and the absorption of light by the LSPR and interband transitions in $\mathrm{Au}$ nanoparticles becomes dominant. Nevertheless, the interference features are damped but not rubbed out after successive infiltrations, showing that light does still travel through the film thickness and that the material remains at least partially transparent in this spectral range.

The data were analyzed using both the DeltaPsi2 software from Horiba Scientific and the Complete Ease Software from JA Woolam Company. SE data measured on the bare silicon substrate were analyzed using the $\mathrm{Si}$ and $\mathrm{SiO}_{2}$ tabulated dielectric functions and yielded a thickness value $(2.0 \mathrm{~nm})$ for the native silica layer on the surface, which was fixed in the further analysis. SE of the PVP-PS diblock copolymer film, before gold introduction, gave the initial thickness, and demonstrated a finite amount of depolarization quantified by $P D=1-\sqrt{I_{s}{ }^{2}+I_{c}{ }^{2}+I_{c}{ }^{2}}$, which could be well modeled as a thickness non-uniformity. The latter non-uniformity was estimated to be close to $8 \%$ and kept fixed 
through-out the fits. One of the possible causes for this thickness non-uniformity is the presence of thickness defects observed on block copolymer thin films when the total thickness of the film is not exactly commensurate with the lamellar period: $\mathrm{T}=n \mathrm{~d}_{\mathrm{o}}$ or $\mathrm{T}=(n+1 / 2) \mathrm{d}_{\mathrm{o}}$, where $n$, a small integer, is the number of complete bilayers ${ }^{52,39,53,54}$.

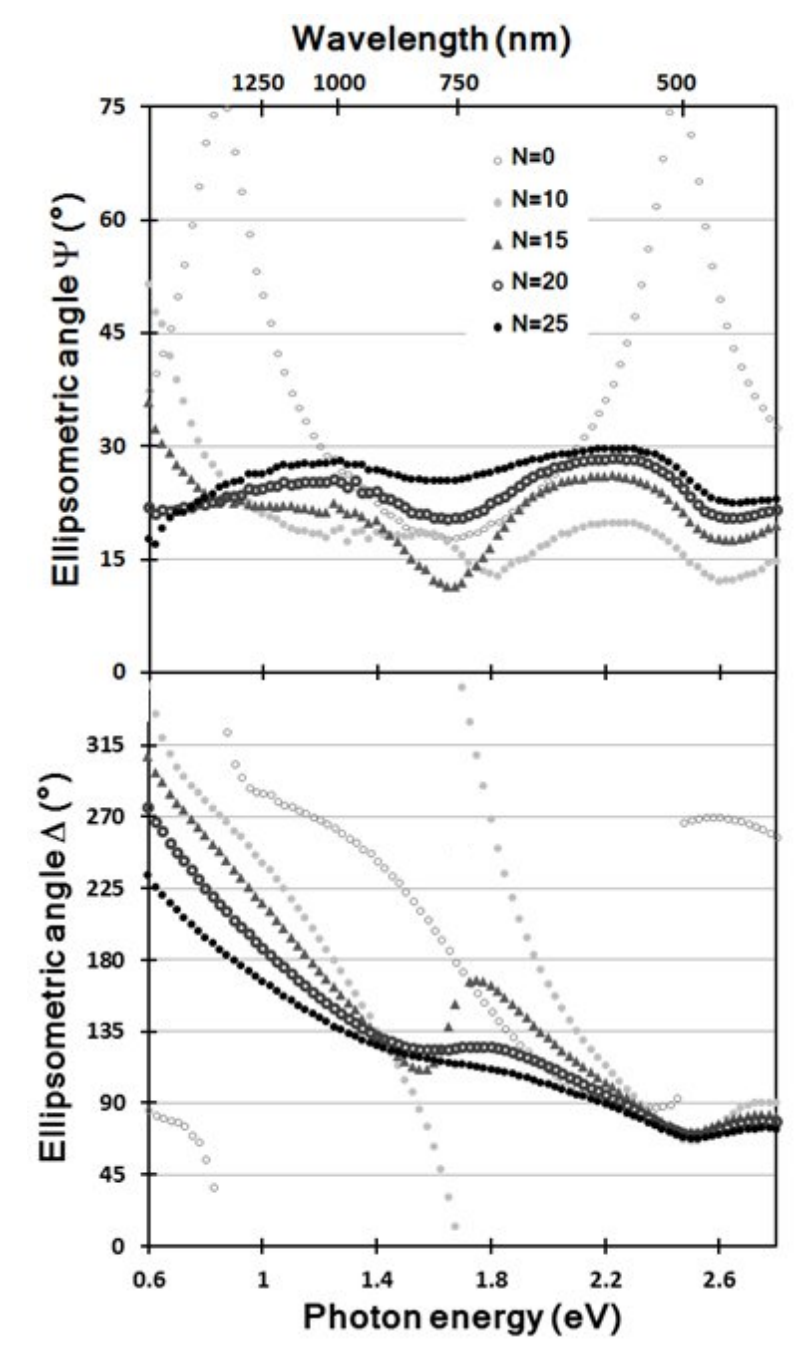

Figure 4. Evolution of the measured ellipsometric angles $\Psi$ and $\Delta$ as a function of the photon energy for an angle of incidence of $\theta_{0}=65^{\circ}$ and different values of $\mathrm{N}$ between 0 and 25 . The spectroscopic features are damped as $\mathrm{N}$ increases, due to the increasing absorption of the film.

\section{II.2 Ellipsometric modelling.}

In order to extract the effective optical properties of the studied films from the SE data, two different models were built (see Figure 4). Due to the small sizes with respect to wavelength of both the gold NPs $D \sim 0.02 \lambda$ and the copolymer nanostructure lamellae $\mathrm{d}_{\mathrm{o}} \sim 0.05 \lambda$ at $\lambda=500 \mathrm{~nm}$, an effective medium (EM) description is appropriate, for all or part of the multilayer stack. The models are built using the information on the layer thicknesses, appropriate interface layers and number of bi-layer 
repetitions inferred from the SEM images and schematized in Figure 5.a. The N-dependent dielectric functions of the polymer-gold nanocomposite effective media NC and EM2 were "kinetically" analyzed, meaning in consistency with a progressive evolution with increasing $\mathrm{N}$, in order to refine the initial guesses.

(a)

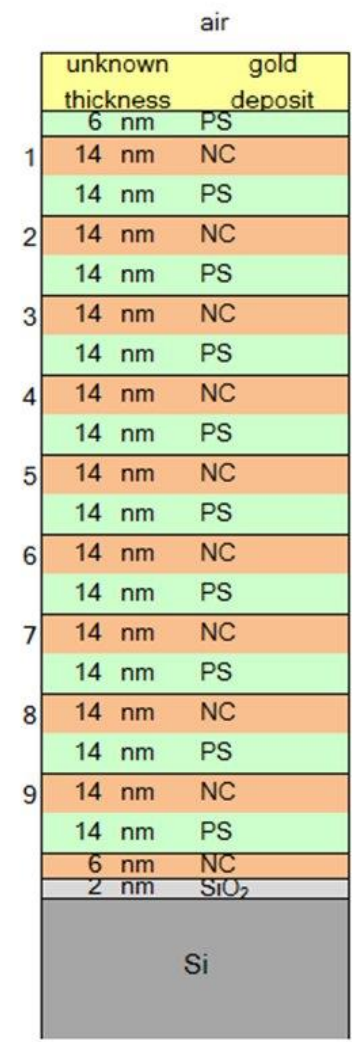

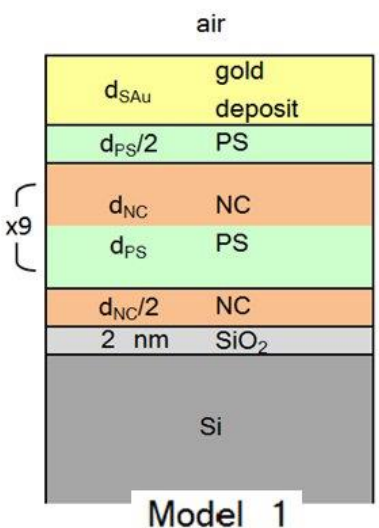

(b)

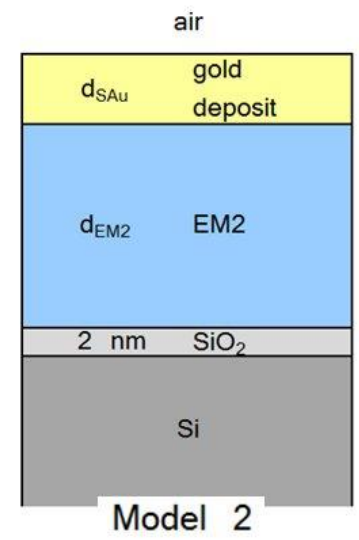

(c)

Figure 5. The models are built based on the detailed film nanostructure obtained from the cross-section SEM images, as schematized in (a). The ellipsometric models used in Models 1 and 2 are represented in (b) and (c), respectively. PS stands for pure polystyrene and $\mathrm{NC}$ for the nanocomposite composed of gold nanoparticles and poly(2-vinylpyridine), of varying composition when $\mathrm{N}$ increases. The thickness of the native silica layer covering the silicon wafer was determined by VASE on a bare wafer. The "kinetic" SE data are analyzed so as to extract the $\mathrm{N}$-independent dimensions $\mathrm{d}_{\mathrm{NC}}, \mathrm{d}_{\mathrm{PS}}, \mathrm{d}_{\mathrm{EM} 2}$, and the $\mathrm{N}$-dependent parameters, which are the thickness $d_{S A u}$ and dielectric function of the upper gold layer, and the dielectric functions of the polymer-gold nanocomposite effective media NC, and EM2.

The stack structure can be schematized as shown on Figure 5.a. In the Model 1 (Figure 5.b), a perfect periodic superlattice is considered to determine the optical properties of the individual layers of the stack. Although this multilayer ellipsometric model does not provide a direct access to the anisotropic effective permittivity of the material, it is used as an intermediate step, from which an effective uniaxial dielectric function will be derived in a second step, as will be explained below. As seen on Figure 5.b, the thickness of the individual layers are $\mathrm{d}_{\mathrm{PS}}, \mathrm{d}_{\mathrm{NC}}$, where the bottom and top half-layer 
thickness are fixed to $\mathrm{d}_{\mathrm{PS}} / 2$ and $\mathrm{d}_{\mathrm{NC}} / 2$, respectively, as usually observed in lamellar block copolymer thin films ${ }^{51}$. An additional homogeneous layer of thickness $d_{\mathrm{SAu}}$ is added in the model, to account for the uncontrolled gold deposit layer. The optical properties of the PS layer are fixed to those of the Woolam database. The permittivity $\varepsilon_{\mathrm{NC}}$ of the NC layers is determined by fitting the Model 1 to the SE data using the BSPLINE function available in the Complete Ease software and using a MaxwellGarnett Effective medium as an initial guess. This fitting procedure allows to extract $\varepsilon_{\mathrm{NC}}(\lambda)$ for the different values of $\mathrm{N}$.

In order to access the anisotropic effective permittivity of the lamellar material, we then apply an effective medium approximation to the perfect periodic lattice of Model 1. It is well known that the transfer matrix for the superlattice, $\mathrm{M}_{\mathrm{SL}}=\left(\mathrm{M}_{\mathrm{PS}} \times \mathrm{M}_{\mathrm{NC}}\right)^{m}$ may be replaced by the transfer matrix of an effective uniaxial layer $\left(\mathrm{M}_{\text {uniaxial }}\right)$ if the number of repetitions $m$ is large, and $\frac{2 \pi}{\lambda_{o}} n_{P S} d_{P S} \ll<1$ and $\mathrm{n}$ $\frac{2 \pi}{\lambda_{o}} n_{N C} d_{N C} \ll<1$, where $\mathrm{n}_{\mathrm{PS}}$ and $\mathrm{n}_{\mathrm{NC}}$ are the optical indices of PS and NC, respectively, the latter being a function of $\mathrm{N}$. If the conditions are not fulfilled, the transfer matrices do not have a unique solution at several angles of incidence simultaneously, and the stack cannot be represented by a simple uniaxial effective medium (basically the diagonal elements of the $\mathrm{M}_{\mathrm{SL}}$ transfer matrix become different ${ }^{55}$ ).

When the three mentioned conditions are fulfilled, the optical properties of the uniaxial effective medium $\varepsilon_{/ /}(\lambda)=\varepsilon_{/ /}{ }^{\prime}(\lambda)+\mathrm{i} \varepsilon_{/ /}{ }^{\prime}(\lambda)$ and $\varepsilon_{\mathrm{z}}(\lambda)=\varepsilon_{\mathrm{z}}{ }^{\prime}(\lambda)+\mathrm{i} \varepsilon_{\mathrm{z}}{ }^{\prime}(\lambda)$ can be written ${ }^{56,57,58}$ :

$$
\begin{array}{ll}
\varepsilon_{/ /}=\left(\frac{d_{N C}}{d_{N C}+d_{P S}}\right) \varepsilon_{N C}+\left(\frac{d_{P S}}{d_{N C}+d_{P S}}\right) \varepsilon_{P S} & \text { Equation 4 } \\
\frac{1}{\varepsilon_{z}}=\left(\frac{d_{N C}}{d_{N C}+d_{P S}}\right) \frac{1}{\varepsilon_{N C}}+\left(\frac{d_{P S}}{d_{N C}+d_{P S}}\right) \frac{1}{\varepsilon_{P S}} & \text { Equation 5 }
\end{array}
$$

As was explained in §I., the condition of deeply subwavelength thicknesses was one of our requirements for the design of a hyperbolic medium. With $\mathrm{d}_{\mathrm{NC}} \sim \mathrm{d}_{\mathrm{PS}}<0.04 \lambda$ on the explored wavelength range, these conditions appear valid even at the resonance, where we expect that $\mathrm{n}_{\mathrm{NC}}$ can be large $e^{45,59}$. Moreover, the number of repetitions is in our case $m=9$, which we regard as sufficient, in particular in view of the absorption in the layers ${ }^{60}$. Finally, Model 1 first allows us to extract $\varepsilon_{\mathrm{NC}}$ from the experimental VASE data, from which we then calculate $\varepsilon_{/ /}$and $\varepsilon_{\mathrm{z}}$, using Equations 4 and 5.

The Model 2 (Figure 5.c) is a direct uniaxial effective medium approach (compared to the indirect approach using the fitted lamellar stack) in which we use the super-lattice results as initial guesses. It combines the surrounding half layers, i.e. the bottom $d_{N C} / 2$ and top layer $d_{P S} / 2$ into the effective uniaxial layer, in order to account for the whole self-assembled diblock copolymer film. The uniaxial permittivity $\left(\varepsilon_{/ /}, \varepsilon_{\mathrm{z}}\right)$ of the medium EM2 is determined by fitting the Model 2 to the SE data using the 
BSPLINE function available in the Complete Ease software and using the results of Model 1 as an initial guess. This fitting procedure allows to extract $\varepsilon_{/ /}(\lambda)$ and $\varepsilon_{z}(\lambda)$ for the different values of $N$.

\section{II.3 Results}

Let us now present and discuss the optical parameters that were extracted from the ellipsometric study for the fabricated nanostructures.

\section{A/ Nanocomposite (NC) layers}

We start by extracting the optical properties of the individual NC layers which make up the lamellar stack. The NC dielectric functions are accessible via Model 1 (Figure 5.b.). The uncontrolled gold deposit layer cannot be represented by a simple effective medium with a few parameters only and was analyzed using a free BSPLINE. Using the kinetic SE data, the fit converged to a top layer dominated by a resonance near a photon energy of $1 \mathrm{eV}(\lambda=1240 \mathrm{~nm})$, which is typical of a partially connected network of Au particles on the surface. The thickness of this Au pollution layer was found to increase from $4 \mathrm{~nm}$ at $\mathrm{N}=5$ to $20 \mathrm{~nm}$ at $\mathrm{N}=25$, which is in agreement with the SEM observations. In fact, for $\mathrm{N} \leq 15$, the results show very little variation when removing the top layer from the model $\left(\mathrm{d}_{\mathrm{SAu}}=0\right)$, confirming its presence is negligible.

Finally, the resulting dielectric function $\varepsilon_{\mathrm{NC}}(\lambda)=\varepsilon_{\mathrm{NC}}{ }^{\prime}(\lambda)+\mathrm{i} \varepsilon_{\mathrm{NC}}{ }^{\prime \prime}(\lambda)$ for the NC layers in the superlattice, from $\mathrm{N}=5$ to $\mathrm{N}=30$, are shown on Figure 6. The optical properties of the Au-loaded polymer NC layer are dominated by a resonance at $\lambda=580 \mathrm{~nm}$, close to that expected ${ }^{45}$ for the plasmon resonance of the gold NPs present in the NC layers, with an amplitude increasing with the number of loading cycles $\mathrm{N}$, as expected. In particular, the NC medium presents a pseudo-metallic behavior beyond $\mathrm{N}=15$ with $\varepsilon_{\mathrm{NC}}$ ' $<0$ on a large wavelength range, of width $80 \mathrm{~nm}$ for $\mathrm{N}=25$ and $50 \mathrm{~nm}$ for $\mathrm{N}=20$. This "pseudo-metal" behavior being resonant in nature is associated with a significant level of losses as can also be seen on Figure 6.

The resulting dielectric functions are consistent with what can be expected for a disordered composite of spherical inclusions within a homogeneous matrix. In fact, such composite can be described, at least at small gold volume fraction by the Maxwell-Garnett effective medium function ${ }^{61}$, written:

$$
\varepsilon_{M G}=\varepsilon_{m}+3 f \varepsilon_{m} \frac{\varepsilon_{A u}-\varepsilon_{m}}{\varepsilon_{A u}+2 \varepsilon_{m}-f\left(\varepsilon_{A u}-\varepsilon_{m}\right)} \quad \text { Equation } 6
$$

where $\varepsilon_{\mathrm{MG}}$ is the effective permittivity of the gold NP-polymer composite, $\varepsilon_{\mathrm{m}}$ is the polymer matrix permittivity, $\varepsilon_{\mathrm{Au}}$ is the NP gold permittivity, and $f$ is the gold volume fraction in the nanocomposite NC. Figure 7 shows how the extracted values of $\varepsilon_{\mathrm{NC}}$ compare with those of $\varepsilon_{\mathrm{MG}}$, calculated using 
Equation 6 and a dielectric function for gold modified from the Johnson \& Christy tabulated data ${ }^{62}$ in order to take into account finite size effects (see reference 45). All values of $\varepsilon_{\mathrm{m}}$ and $\varepsilon_{\mathrm{Au}}$ used in the $\varepsilon_{\mathrm{MG}}$ calculation are displayed in the Supplementary Information, Figure S2.

For small $\mathrm{N} \leq 5$ (small gold fraction), the agreement is very good on all the red part of the spectrum, for wavelength above the resonance value at $\sim 580 \mathrm{~nm}$, and satisfying at lower wavelength (Figure 7).

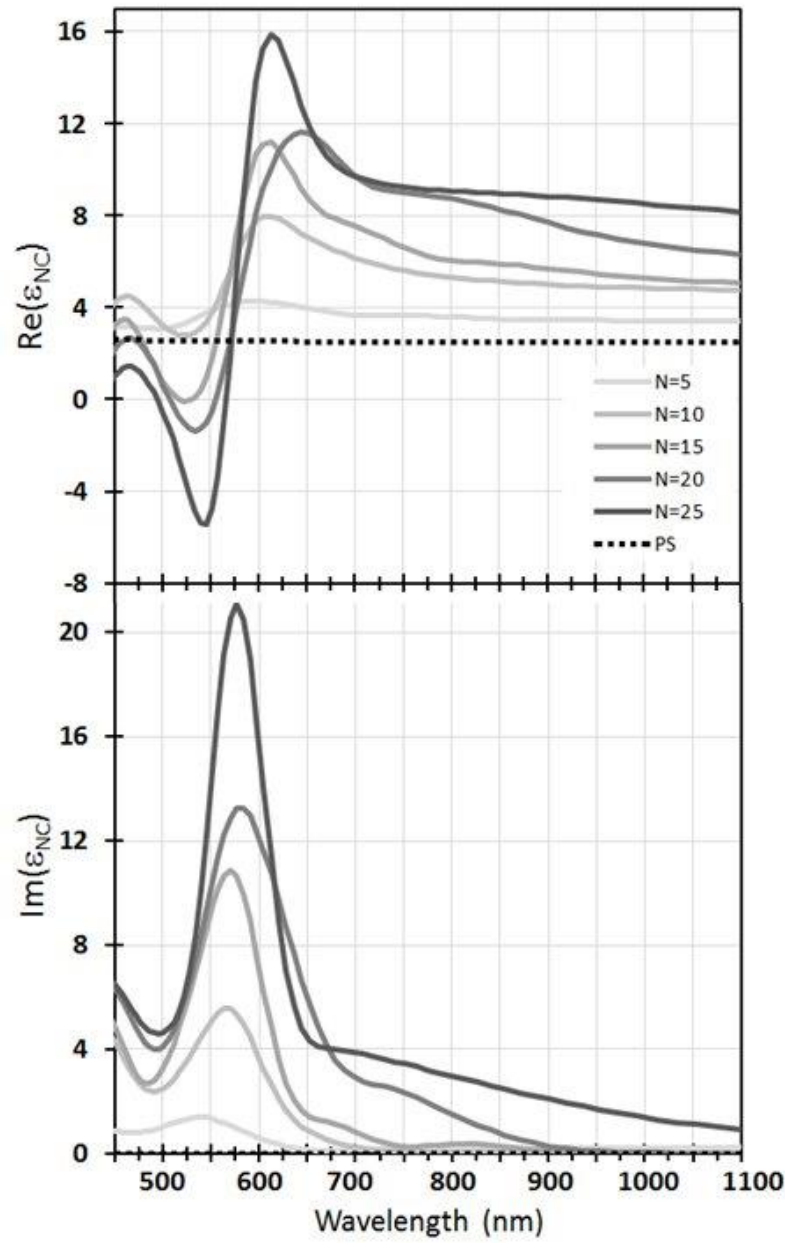

Figure 6. Real (upper plot) and imaginary (lower plot) parts of the dielectric function $\varepsilon_{\mathrm{NC}}$ of the $\mathrm{NC}$ layers in the lamellar stack, as extracted from the SE study for different values of $\mathrm{N}$ between 5 and 25. The dotted line is the dielectric function used for the PS layer, with $\operatorname{Im}\left(\varepsilon_{\mathrm{PS}}\right)=0$. The resonance amplitude varies as $\mathrm{N}$ increases, due to the increasing introduction of plasmonic NPs.

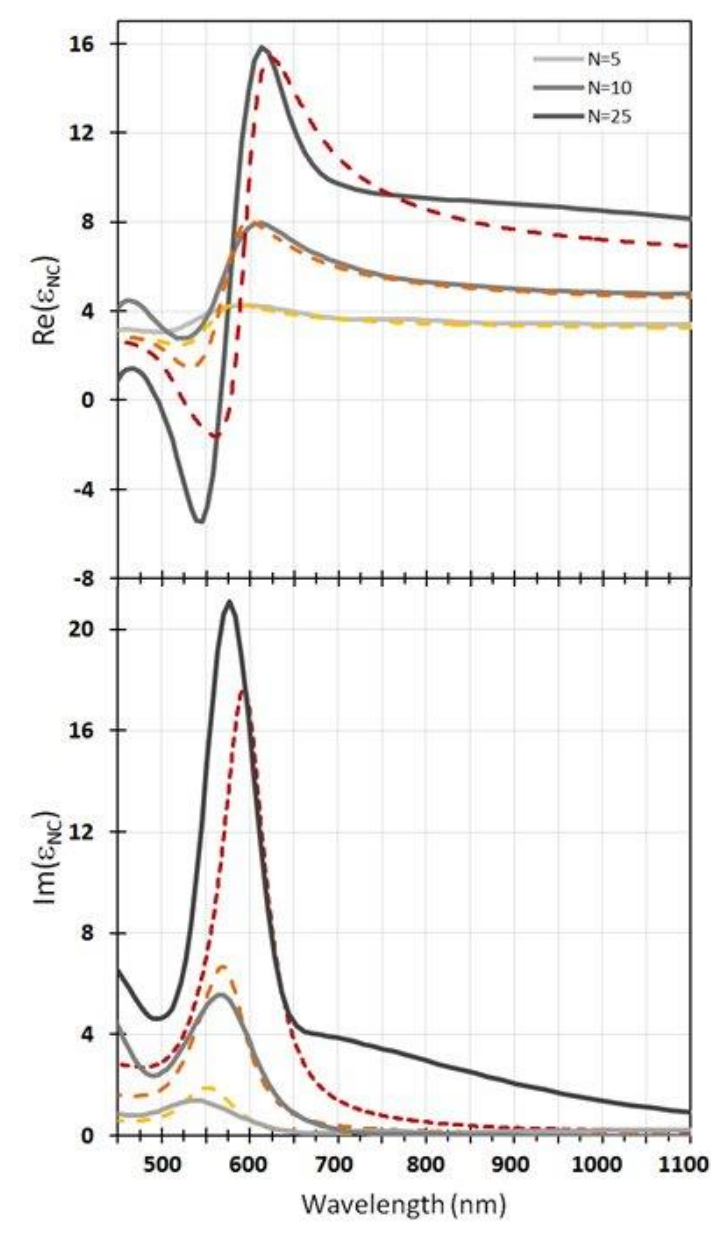

Figure 7. Comparison between the real (upper plot) and imaginary (lower plot) parts of the dielectric function $\varepsilon_{\mathrm{NC}}$ of the NC layers in the lamellar stack, as extracted from the SE study (solid lines), and the values calculated as a Maxwell-Garnett effective medium $\varepsilon_{\mathrm{MG}}$ (dashed lines) for different volume fractions in gold: $f=7 \%$ (yellow line), $18 \%$ (orange line), $31 \%$ (red line).

When pushing the Maxwell-Garnett effective medium approximation (MG-EMA) to higher N, a degraded agreement is naturally expected: it remains nevertheless reasonably good especially above $580 \mathrm{~nm}$. These partial agreements provide rough estimates of the loading concentration in gold: we 
find $\mathrm{N}=5$ to correspond to a MG-extracted value of $f=7 \%$, and $\mathrm{N}=10$ and $\mathrm{N}=25$ to approximately $f$ $=18 \%$ and $f=31 \%$, respectively.

\section{B/ Uniaxial effective medium}

Following the second step of Model 1, the dielectric constants of the uniaxial effective medium $\varepsilon_{/ /}(\lambda)=$ $\varepsilon_{/ /}{ }^{\prime}(\lambda)+\mathrm{i} \varepsilon_{/ /}{ }^{\prime \prime}(\lambda)$ and $\varepsilon_{\mathrm{z}}(\lambda)=\varepsilon_{\mathrm{z}}{ }^{\prime}(\lambda)+\mathrm{i} \varepsilon_{\mathrm{z}}{ }^{\prime \prime}(\lambda)$ are first determined using the optical properties of the fitted $\mathrm{Au}$ loaded polymer layers (NC) and the PS layer, through Equations 4 and 5. The resulting effective optical properties are shown in Figure 8.
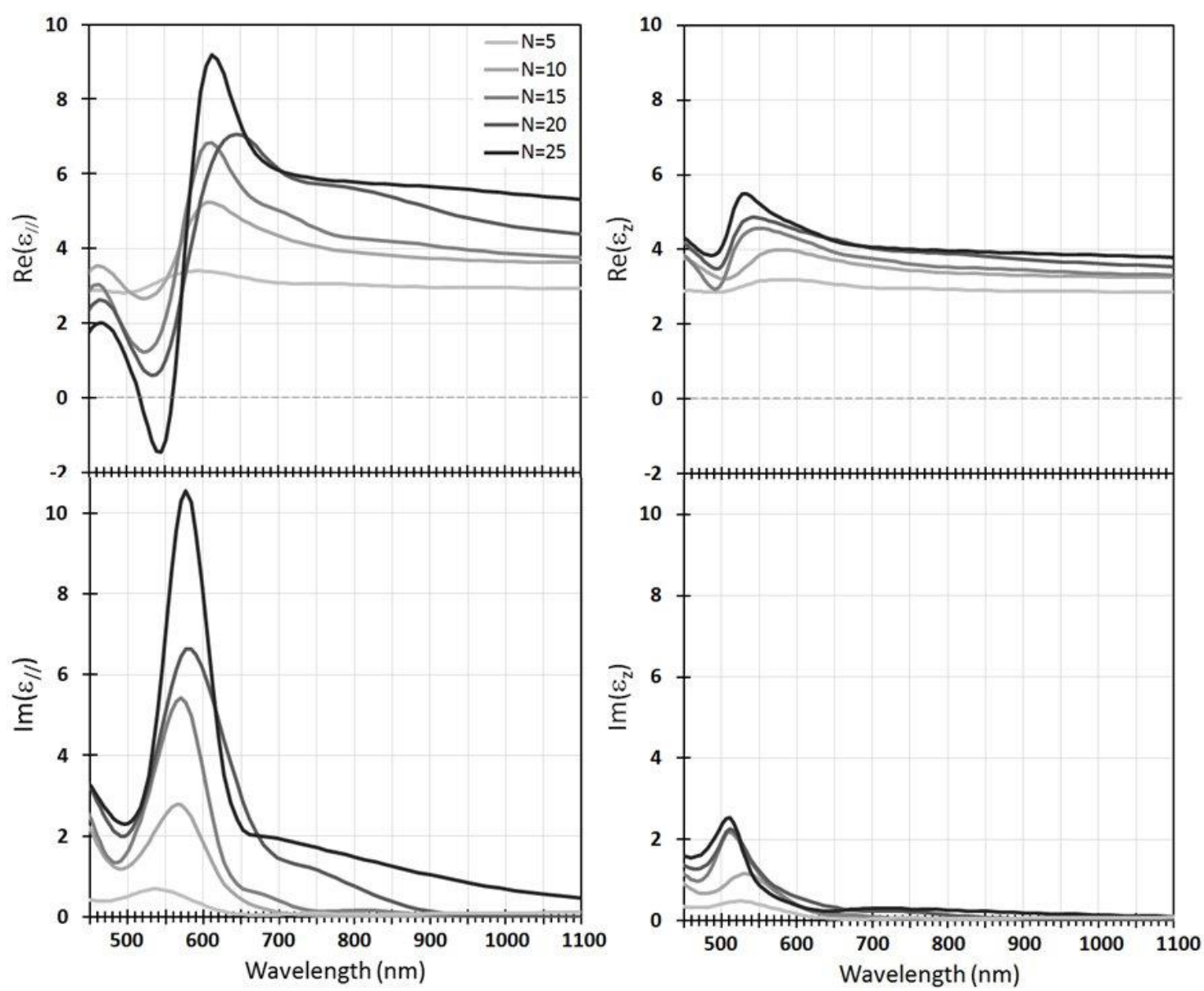

Figure 8. Real (upper plots) and imaginary (lower plots) parts of the components $\varepsilon_{/ /}$(left) and $\varepsilon_{\mathrm{z}}$ (right) of the lamellar nanoplasmonic thin films, as computer using Equations 4 and 5 from the Model $1 \mathrm{SE}$ extractions for different values of $\mathrm{N}$ between 5 and 25 (MGextracted $f$ between 7 and $31 \%$,). The resonance amplitude varies as $\mathrm{N}$ increases, due to the increasing volume fraction of introduced plasmonic nanoparticles.

The analysis of the SE data through direct inversion as a uniaxial effective medium (Model 2) leads to a similar or better goodness-of-fit, compared to Model 1. Figure 9 shows the comparison between the components $\varepsilon_{/ /}{ }^{\prime}(\lambda)$ extracted for $\mathrm{N}=15, \mathrm{~N}=20$ and $\mathrm{N}=25$ using Models 1 and 2. Models 1 and 2 were found to give highly similar results, within the experimental accuracy, in particular for $\varepsilon_{/ /}$, 
although $\varepsilon_{\mathrm{Z}}$ shows a little more variation between the models. The agreement between the extractions of the two models substantiates the main result of this experimental study showing that the dielectric functions $\varepsilon_{/ /}$and $\varepsilon_{\mathrm{z}}$ have opposite signs in a finite wavelength range.

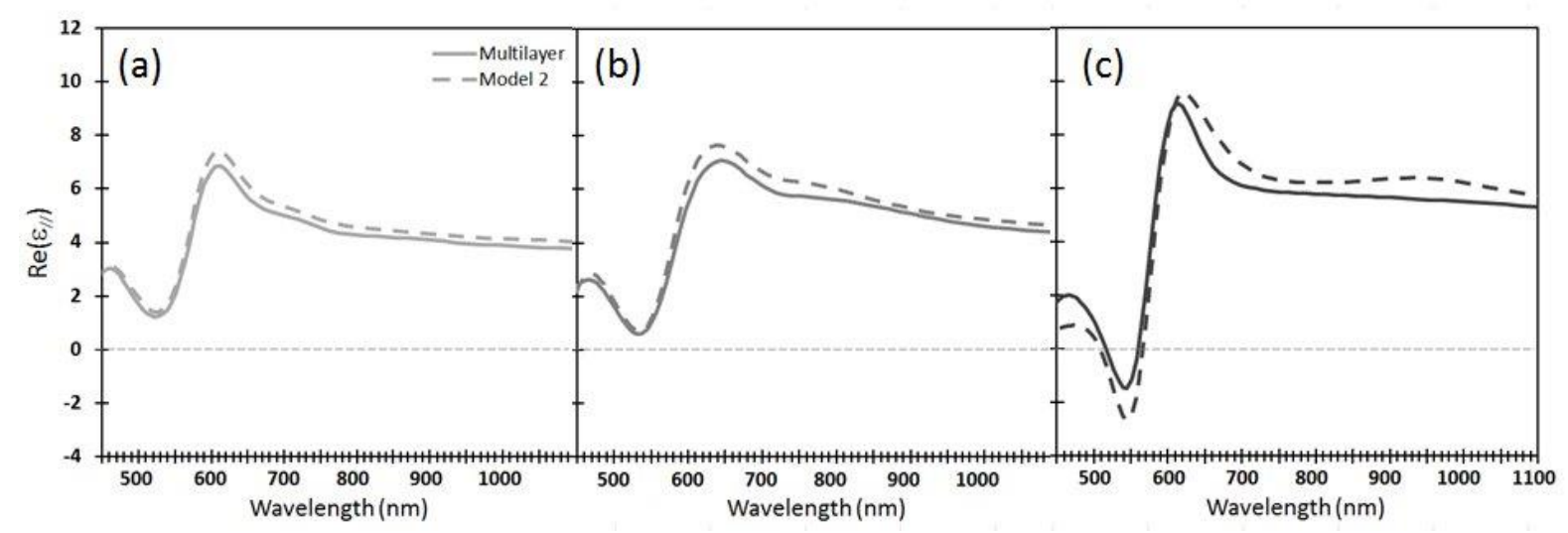

Figure 9. Comparison between the real part of the ordinary components in the cases of the multilayer (Model 1, continuous lines) and uniaxial model (Model 2, dashed lines) for $\mathrm{N}=15$ (a), $\mathrm{N}=20$ (b) and $\mathrm{N}=25$ (c).

As is shown on the Figure 8, the dielectric functions $\varepsilon_{/ /}$and $\varepsilon_{\mathrm{z}}$ both present a resonance at the wavelength $\lambda=580 \mathrm{~nm}$ (or alternatively a photon energy of $2.1 \mathrm{eV}$ ), close to the plasmon resonance of the gold nanoparticles. However, the resonance amplitudes of the two components significantly differ. In the case of $\varepsilon_{z}$, this amplitude is limited and varies only little with the value of $\mathrm{N}$, in direct relation with the amount of gold nanoparticles in the nanostructure. By contrast, the resonance of $\varepsilon_{/ /}$is stronger and its amplitude significantly increases with $\mathrm{N}$, reaching a regime of negative values beyond $\mathrm{N}=20$. As a result, strong artificial anisotropies with different characteristics are found across the spectrum.

Finally note that attempting to combine the top Au deposit layer (of thickness $d_{\mathrm{SAu}}$ ) into the effective uniaxial medium smears its properties and does not produce negative $\varepsilon_{/ /}$, in contrast with the analyses based on the two used models. Therefore, future studies will aim at lifting off the uncontrolled detrimental gold deposit top layer. In the current analysis, it is regarded as an experimental artifact whose analysis needs to be isolated from the analysis of the targeted nanostructure below it.

\section{C/ Hyperbolic dispersion relation}

We consider three regions of strong anisotropy denoted A: $\varepsilon_{\mathrm{z}}{ }^{\prime}>\varepsilon_{/ /}{ }^{\prime}>0(\lambda=481 \mathrm{~nm})$, B: $\varepsilon_{/ /}{ }^{\prime}<0<\varepsilon_{\mathrm{z}}{ }^{\prime}$ $(\lambda=539 \mathrm{~nm})$ and $\mathrm{C}: \varepsilon_{/ /}{ }^{\prime}>\varepsilon_{\mathrm{z}}{ }^{\prime}>0(\lambda=670 \mathrm{~nm})$ indicated on Figure 10, which displays the values of $\varepsilon_{/ /}$ and $\varepsilon_{z}$ for $\mathrm{N}=25$ (corresponding to a MG-extracted $f=31 \%$ ). The iso-frequency dispersion diagrams of the constitutive material can be produced using the experimental dielectric functions and are also 
shown on Figure 10. For each frequency $\omega$ considered (or equivalently, excitation wavelength), two wavevector ranges can be distinguished $\left|k_{\mathrm{x}}\right|<k_{\mathrm{x}}^{*}$ and $\left|k_{\mathrm{x}}\right|>k_{\mathrm{x}}^{*}$, with

$$
k_{x}^{*}=k_{o} \sqrt{\frac{\varepsilon_{z}{ }^{2}+\varepsilon_{z}{ }^{\prime 2}}{\varepsilon_{z}{ }^{\prime}}} \quad \text { Equation 7 }
$$

and $k_{\mathrm{o}}{ }^{2}=\varepsilon_{\mathrm{o}} \mu_{\mathrm{o}} \omega^{2}$.
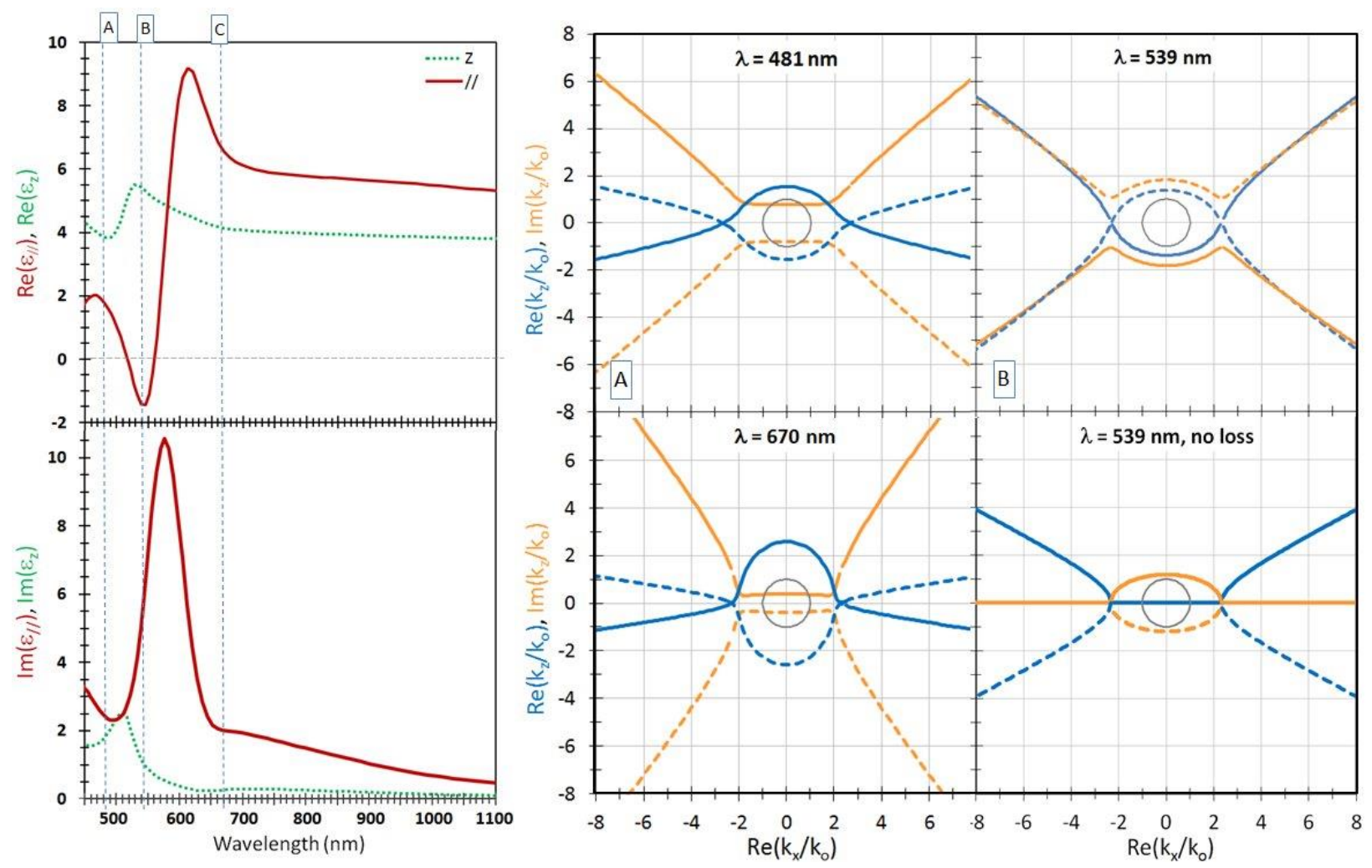

Figure 10. Left: Parallel (red continuous line) and perpendicular (green dotted line) components of the uniaxial dielectric function of the lamellar nanoplasmonic stack for $\mathrm{N}=25\left(\mathrm{f}_{\mathrm{Au}} \sim 30 \%\right.$ in the $\mathrm{NC}$ layers). The vertical blue dashed lines indicate the three conditions (A: $\lambda=481 \mathrm{~nm} ; \mathrm{B}: \lambda=539 \mathrm{~nm} ; \mathrm{C}: \lambda=670 \mathrm{~nm}$ ) chosen for the calculation of the dispersion relations, which are displayed in the right part. Right: Dispersion relation at $k_{\mathrm{y}}=0$, showing the real (blue lines) and imaginary (orange lines) parts of the $k_{\mathrm{z}}$ component of the wave-vectors in the material as a function of the $k_{\mathrm{x}}$ component. Thick continuous and dashed lines correspond to opposite solutions of Equation 3. The thin black continuous circle corresponds to the wavevectors propagating in air $k_{x}^{2}+k_{z}^{2}=k_{o}^{2}$, shown for reference. The plot labeled $\mathrm{D}$, shown for reference, is a fictional hyperbolic case without losses: at $\lambda=539$ $\mathrm{nm}, \varepsilon_{/ /}$' and $\varepsilon_{\mathrm{z}}{ }^{\prime}$ are set to the values for case $\mathrm{B}$, whereas $\varepsilon_{/ /}$' and $\varepsilon_{\mathrm{z}}$ " are set to zero.

Cases $\mathrm{A}$ and $\mathrm{C}$ present typical elliptical dispersion diagrams, when both $\varepsilon_{/ /}{ }^{\prime}$ and $\varepsilon_{\mathrm{z}}{ }^{\prime}$ are positive: one can observe, for small values of $\left|k_{\mathrm{x}}\right|\left(\left|k_{\mathrm{x}}\right|<k_{\mathrm{x}}^{*}\right)$, two symmetrical elliptical branches where the real part of $k_{\mathrm{z}}$ is significantly larger than the imaginary part, typical of an attenuated propagation. For larger $\left|k_{\mathrm{x}}\right|$ $\left(\left|k_{\mathrm{x}}\right|>k_{\mathrm{x}}{ }^{*}\right)$, hyperbolic branches are observed, which are not usually present in ideal elliptical cases, 
and which are characterized by a predominantly evanescent behavior (imaginary part of $k_{\mathrm{z}}$ much larger than the real part). In the case B when $\varepsilon_{/ /},<0<\varepsilon_{\mathrm{z}}$, we find that the dispersion diagram present hyperbolic branches for $\left|k_{\mathrm{x}}\right|>k_{\mathrm{x}}^{*}$, as well as elliptical curves at small $\left|k_{\mathrm{x}}\right|$.

These diagrams are related to the strong optical anisotropy of the lamellar stack, obtained as a consequence of the resonant nature of the NC layers. The significant losses $\left(\operatorname{Re}\left(k_{\mathrm{z}}\right) \approx \operatorname{Im}\left(k_{\mathrm{z}}\right)\right)$ associated with the hyperbolic propagation are another consequence of this resonant nature.

To understand the significance of these dispersion curves, it is useful to recall the usual description of elliptical and hyperbolic media. The ideal cases are displayed in the Figures 11.a and 11.d, where two fictitious materials are considered with the real parts of the permittivity tensor set to the experimentally determined values in cases $\mathrm{C}\left(\varepsilon_{/ /}{ }^{\prime}=1.77, \varepsilon_{\mathrm{z}}{ }^{\prime}=3.85\right)$ and $\mathrm{B}\left(\varepsilon_{/ /}{ }^{\prime}=-1.42, \varepsilon_{\mathrm{z}}{ }^{\prime}=5.42\right)$; and with imaginary parts set to zero: $\varepsilon_{/ /} "=\varepsilon_{\mathrm{z}} "=0$ (lossless materials). We represent the dispersion relation (Equation 3) in the $k_{\mathrm{y}}=0$ plane. In the elliptical ideal case (Fig. 11.a), the propagative modes $\left(\operatorname{Re}\left(k_{\mathrm{z}}\right) \neq 0\right)$ are restricted to small wavenumbers $\left(\left|k_{\mathrm{x}}\right|<k_{\mathrm{x}}{ }^{*}\right)$, while all possible large- $\left|k_{\mathrm{x}}\right|$ modes are forbidden ${ }^{63}$ and only purely evanescent $\left(\operatorname{Re}\left(k_{z}\right)=0\right)$. In the hyperbolic ideal case (Fig. 11.d), we observe the opposite situation, with forbidden, purely evanescent modes $\left(\operatorname{Re}\left(k_{\mathrm{z}}\right)=0\right)$ for small $\left|k_{\mathrm{x}}\right|$ and purely propagative modes $\left(\operatorname{Re}\left(k_{\mathrm{z}}\right) \neq 0, \operatorname{Im}\left(k_{\mathrm{z}}\right)=0\right)$ for large $\left|k_{\mathrm{x}}\right|$, the latter potentially providing the super-resolution property. The hyperbolic branches for $\left|k_{\mathrm{x}}\right|>k_{\mathrm{x}}{ }^{*}$ present the shape of a one-fold hyperboloid, with a forbidden gap in $k_{\mathrm{x}}$, as expected for a "metallic" ${ }^{64}$ or type $\mathrm{II}^{4}$ hyperbolic medium, when the permittivity tensor $\underline{\underline{\varepsilon}}$ has two negative and one positive components.

To depart from these idealized situations, let us now introduce a small amount of losses in the materials, see Figure 11.b and 11.e (here we arbitrarily took $\varepsilon_{/ /} "=4 \varepsilon_{\mathrm{z}}{ }^{\prime}=0.8$ ): the dispersion curves become hybrid rather than purely elliptical or hyperbolic and present finite $\operatorname{Re}\left(k_{\mathrm{z}}\right)$ and $\operatorname{Im}\left(k_{\mathrm{z}}\right)$ at all values of $k_{\mathrm{x}}$, allowing previously forbidden modes to propagate weakly. Purely evanescent waves for the hyperbolic medium at small $\left|k_{\mathrm{x}}\right|$ and the elliptical medium at large $\left|k_{\mathrm{x}}\right|$ become weakly propagative with $\operatorname{Re}\left(k_{\mathrm{z}}\right) \neq 0$ and $\operatorname{Im}\left(k_{\mathrm{z}}\right)>>\operatorname{Re}\left(k_{\mathrm{z}}\right)$. The large- $\left|k_{\mathrm{x}}\right|$ branches of the hyperbolic medium (Fig. 11.f) present the same one-fold hyperboloid shape as that of the no-loss case (Fig. 11.e). Interestingly, when the imaginary parts $\varepsilon_{/ /}$" and $\varepsilon_{\mathrm{z}}$ " are further increased to simulate more losses (Fig. 11.(c-d) and (g-h)), the modification of the propagative and evanescent modes continues to occur, so much so that over most of the whole $k_{\mathrm{x}}$ range, more or less strongly attenuated, propagative modes are observable. In particular, we note that the intermediate hyperbolic cases (as represented in Figure 11.f and 11.g) still present predominantly propagative modes at large $\left|k_{\mathrm{x}}\right|$, which can support super-resolution properties: these are the cases corresponding to our experimental system in the B situation in Figure 10. 


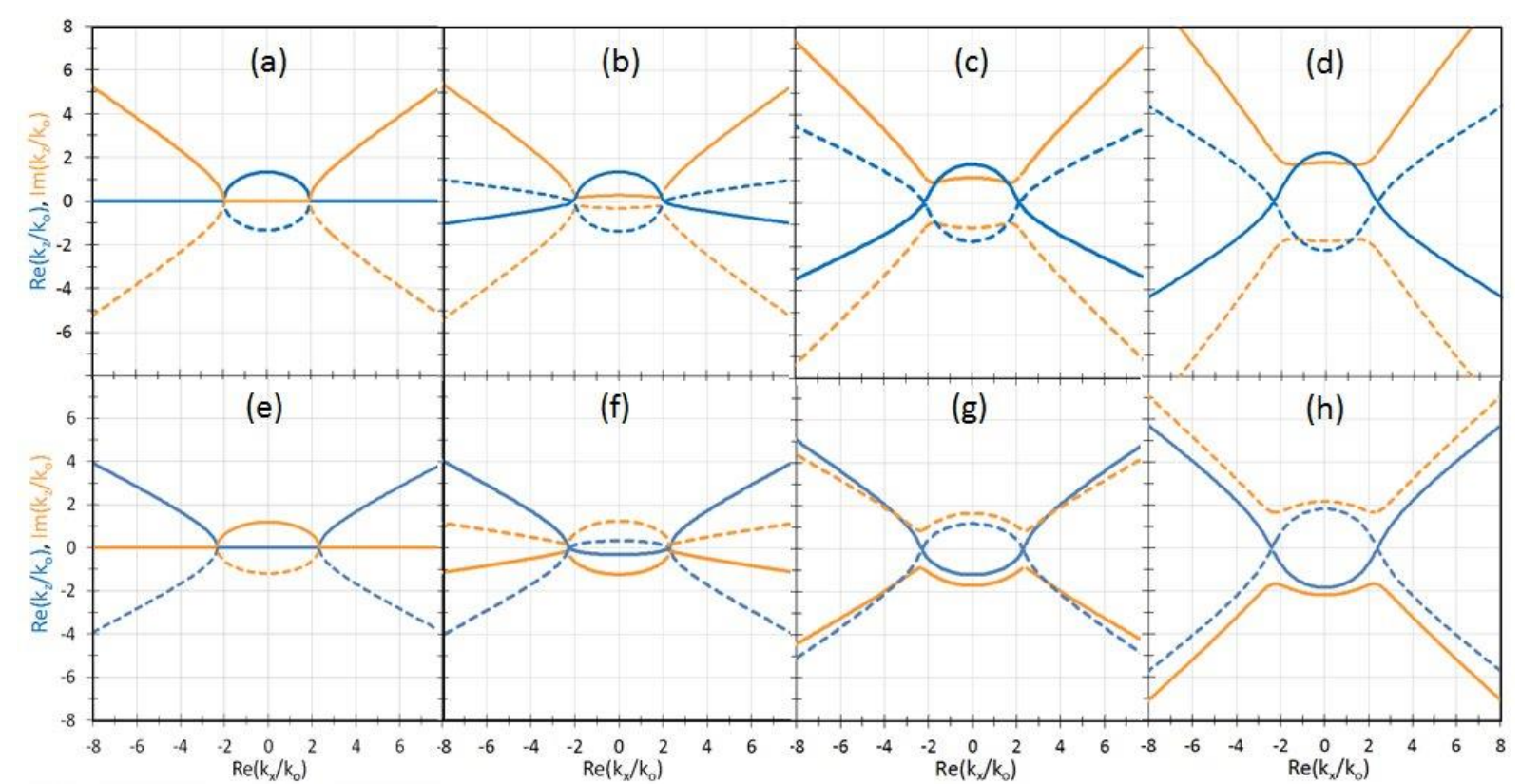

Figure 11. Dispersion relations at $k_{\mathrm{y}}=0$, showing the real (blue line) and imaginary (orange line) parts of the $k_{\mathrm{z}}$ component of the wave-vectors in the material as a function of the $k_{\mathrm{x}}$ component. Continuous and dashed lines correspond to opposite solutions of Equation 3. The plots (a)-(d) (resp. (e)-(h)) correspond to fictional elliptical (resp. hyperbolic) cases with $\varepsilon_{/ /}$' and $\varepsilon_{\mathrm{z}}$ ' set to the values for case $\mathrm{C}$ (resp. B), and $\varepsilon_{/ /} "=4 \varepsilon_{\mathrm{z}}{ }^{\prime \prime}=0$ (a) and (e); $\varepsilon_{/ /} "=4 \varepsilon_{\mathrm{z}} "=0.8$ (b) and (f); $\varepsilon_{/ /} "=4 \varepsilon_{\mathrm{z}} "=4$ (c) and (g); and $\varepsilon_{/ /} "=4 \varepsilon_{\mathrm{z}} "=8$ (d) and (h).

Finally, these results demonstrate the capacity of our bottom-up self-assembled multilamellar stack to respond as a hyperbolic effective medium in a given region of the visible spectrum $(520<\lambda<560$ $\mathrm{nm})$. In the hyperbolic region, the dispersion relation allows propagative modes for large values of $\left|k_{\mathrm{x}}\right|$, potentially providing super-resolution. These hyperbolic modes are however significantly attenuated due to the lossy nature of the media, in which the hyperbolic property is obtained from the resonant nature of the permittivity. These detrimental losses need now to be minimized by tuning the nature, size, size dispersity and organization of the nanoparticles. Furthermore, the same self-assembly methodology for lamellar stacks fabrication could be used with other nanoparticles with a resonance of better quality factor, or with a lamellar system including gain species (fluorophores or quantum dots) which could amplify the plasmons and compensate the losses.

In any case, we have demonstrated for the first time the possibility of using a self-assembly methodology for the fabrication of bulk hyperbolic material, which opens new fabrication routes for metamaterials aiming at super-resolution lenses. 


\section{ACKNOWLEDGMENTS}

We thank I. Ly and C. Picard for help with the SEM imaging, and P. Barois, E. Garcia-Caurel, B. Gallas, and M. Stchakovsky for useful discussions and comments. This work was supported by the LabEx AMADEus (ANR-10-LABX-42) in the framework of IdEx Bordeaux (ANR-10-IDEX-03-02), France. It has benefited from an Aurora Hubert Curien Partnership, funded by the Norges Forskningsraad (NFR) and by the French ministères des Affaires étrangères et du Développement international (MAEDI) and de l'Education nationale l'Enseignement supérieur et de la Recherche (MENESR).

\section{References}

1 J.B. Pendry, D. Schurig, D. R. Smith, Controlling electromagnetic fields, Science 312 (2006) 1780-1782.
2 N. Engheta, Circuits with light at nanoscales: Optical nanocircuits inspired by metamaterials, Science 317
(2007) 1698-1702
${ }^{3}$ B. Wang, K.-M. Huang, Shaping the radiation pattern with mu and epsilon-near-zero metamaterials, Progr. Electromagn. Research. 106 (2010) 107-119

${ }^{4}$ L. Ferrari, C. Wu, D. Lepage, X. Zhang, Z. Liu, Hyperbolic metamaterials and their applications, Progr. Quant. Electr. 40 (2015) 1-40

${ }^{5}$ Y. Guo, W. Newman, C. L. Cortes, Z. Jacob, Applications of Hyperbolic Metamaterial Substrates, Adv. OptoElectr. 452502 (2012) 2012

6 A. Poddubny, I. Iorsh, P. Belov, Y. Kivshar, Hyperbolic metamaterials, Nature Photon. 7 (2013) 958

7 A. A. Orlov, S. V. Zhukovsky, I. V. Iorsh, P. A. Belov, Controlling light with plasmonic multilayers, Photonics Nanostruct. 12 (2014) 213-230

${ }^{8}$ B. Wood, J. B. Pendry, and D. P. Tsai, Directed subwavelength imaging using a layered metal-dielectric system, Phys. Rev. B 74 (2006) 115116

${ }^{9}$ S. V. Shukovsky, O. Kidwai, J. E. Sipe, Physical nature of volume plasmon polaritons in hyperbolic metamaterials, Opt. Exp. 21 (2013) 14982-14987

${ }^{10}$ J. Yao, Z. Liu, Y. Liu, Y. Wang, C. Sun, G. Bartal, A. M. Stacy, X. Zhang, Optical Negative Refraction in Bulk Metamaterials of Nanowires, Science 321 (2008) 930

${ }^{11}$ X. Zhang, Z. Liu, Superlenses to overcome the diffraction limit, Nat. Mat. 7 (2008) 435-441

12 E. E. Narimanov, V.M. Shalaev, Beyond diffraction, Nature 447 (2007) 266-267

${ }^{13}$ K. V. Sreekanth, M. ElKabbash, Y. Alapan, A. R. Rashed, U. A. Gurkan, G. Strangi, A multiband perfect absorber based on hyperbolic metamaterials, Scientific Reports 6 (2016) 26272

${ }^{14}$ H. N. S. Krishnamoorthy, Z. Jacob, E. Narimanov, I. Kretzschmar, V. M. Menon, Topological Transitions in Metamaterials, Science 336 (2012) 205-209

${ }^{15}$ S. N. Kurilkina, M. A. Binhussain, V. N. Belyi, N. S. Kazak, Features of hyperbolic metamaterials with extremal optical characteristics J. Opt. 18 (2016) 085102

${ }^{16}$ C. L. Cortes, W. Newman, S. Molesky, Z. Jacob, Quantum nanophotonics using hyperbolic metamaterials, J. Opt. (2012) 063001

${ }^{17}$ Z. Jacob, J.-Y. Kim, G.V. Naik, A. Boltasseva, E.E. Narimanov, V.M. Shalaev, Engineering photonic density of states using metamaterials, Appl. Phys. B 100 (2010) 215-218

${ }^{18}$ K. V. Sreekanth, K. H. Krishna, A. De Luca, G. Strangi, Large spontaneous emission rate enhancement in grating coupled hyperbolic metamaterials, Scientific Reports 4 (2014) 6340

${ }^{19}$ Z. Jacob, I. I. Smolyaninov, E. E. Narimanov, Broadband Purcell effect: radiative decay engineering with metamaterials. Appl. Phys. Lett. 100 (2012) 181105. 
${ }^{20}$ D. Lu, J. J. Kan, E. E. Fullerton, Z. Liu, Enhancing spontaneous emission rates of molecules using nanopatterned multilayer hyperbolic metamaterials, Nature Nanotech. 9 (2014) 48-53

${ }^{21}$ S.-A. Biehs, M. Tschikin, P. Ben-Abdallah, Hyperbolic Metamaterials as an Analog of a Blackbody in the Near Field, Phys. Rev. Lett. 109 (2012) 104301

${ }^{22}$ Y. Guo, C. L. Cortes, S. Molesky, Z. Jacob, Broadband super-Planckian thermal emission from hyperbolic metamaterials, Appl. Phys. Lett. 101 (2012) 131106

${ }^{23}$ A. V. Kabashin, P. Evans S. Pastkovsky, W. Hendren, G. A. Wurtz, R. Atkinson, R. Pollard, V. A. Podolskiy, A. V. Zayats, Plasmonic nanorod metamaterials for biosensing. Nat. Mater. 8 (2009) 867-871

${ }^{24}$ K. V. Sreekanth, Y. Alapan, M. ElKabbash, E. Ilker, M. Hinczewski, U. A. Gurkan, A. De Luca and G. Strangi, Extreme sensitivity biosensing platform based on hyperbolic metamaterials, Nat. Materials 15 (2016) 621-627

${ }^{25}$ U. Dürig, D. W. Pohl, F. Rohner, Near-field optical scanning microscopy, J. Appl. Phys. 59 (1986) 3318

${ }^{26}$ T.A. Klar, S. Jakobs, M. Dyba, A. Egner, S. W. Hell, Fluorescence microscopy with diffraction resolution barrier broken by stimulated emission, Proc. Nat. Acad. Sci. 97 (2000) 8206-8210

27 A. N. Boettiger, B. Bintu, J. R. Moffitt, S. Wang, B. J. Beliveau, G. Fudenberg, M. Imakaev, L. A. Mirny, C. $\mathrm{Wu}, \mathrm{X}$. Zhuang, Super-resolution imaging reveals distinct chromatin folding for different epigenetic states, Nature 529 (2016) 418-422

28 T. G. Mackay, Toward optical sensing with hyperbolic metamaterials, Opt. Eng. 54 (2015) 067102

29 J. B. Pendry, Negative Refraction Makes a Perfect Lens, Phys. Rev. Lett. 85 (2000) 3966

${ }^{30}$ C. M. Soukoulis, M. Wegener, Past achievements and future challenges in the development of threedimensional photonic metamaterials, Nat. Photonics 5 (2011) 523-530

${ }^{31}$ T. Xu, A. Agrawal, M. Abashin, K. J. Chau, H. J. Lezec, All-angle negative refraction and active flat lensing of ultraviolet light, Nature 497 (2013) 470-474

32 A. Baron, A. Aradian, V. Ponsinet, P. Barois, Self-Assembled Optical Metamaterials, J. Opt. \& Laser Techn. 82 (2016) 94-100

33 J. Gong, G. Li, Z. Tang, Self-Assembly of Noble Metal Nanocrystals: Fabrication, Optical Property, and Application. Nano Today 7 (2012) 564-585

34 F. S. Bates, G. H. Fredrickson, Block Copolymers-Designer Soft Materials, Physics Today 52 (1999) $32-38$

35 I.W. Hamley, The physics of block copolymers, Oxford Science Publications, 1998

${ }^{36}$ F. S. Bates, G. H. Fredrickson, Block Copolymer Thermodynamics: Theory and Experiment, Annu. Rev. Phys. Chem. 41 (1990) 525-557

37 Y. Fink, A. M. Urbas, M. G. Bawendi, J. D. Joannopoulos, E. L. Thomas, Block Copolymers as Photonic Bandgap Materials, J. Lightwave Technol. 17 (1999) 1963-1969.

38 S. Salvatore, A. Demetriadou, S. Vignolini, S. S. Oh, S. Wuestner, N. A. Yufa, M. Stefik, U. Wiesner, J. J. Baumberg, O. Hess, U. Steiner, Tunable 3D Extended Self- Assembled Gold Metamaterials with Enhanced Light Transmission, Adv. Mater. 25 (2013) 2713-2716

${ }^{39}$ V. Ponsinet, Nanopatterns Produced by Directed Self-Assembly in Block Copolymer Thin Films, in Polymer Surfaces in Motion - Unconventional Patterning Methods, J. Rodríguez-Hernández, C. Drummond, Eds., Springer International Publishing, 2015

40 M. Lazzari, C. De Rosa, Methods for the alignment and the large-scale ordering of block copolymer morphologies, in Block copolymers in nanoscience; M. Lazzari, G. Liu, S. Lecommandoux, Eds, Wiley-VCH, Weinheim, 2006

41 J. G. Kennemur, L. Yao, F. S. Bates, M. A. Hillmyer, Sub-5 nm domains in ordered poly(cyclohexylethylene)-block-poly(methyl methacrylate) block polymers for lithography, Macromolecules 47 (2014) 1411-1418

42 C. Tang, S. Hur, B. C. Stahl, K. Sivanandan, M. Dimitriou, E. Pressly, G. H. Fredrickson, E. J. Kramer, C. J. Hawker, Thin film morphology of block copolymer blends with tunable supramolecular interactions for lithographic applications, Macromolecules 43 (2010) 2880-2889

43 M. Park, C. Harrison, P. M. Chaikin, R. A. Register, D. H. Adamson, Block copolymer lithography: Periodic arrays of similar to 1011 holes in 1 square centimeter, Science 276 (1997) 1401-1404.

${ }^{44}$ M. M. Alam, Y.-R. Lee, J.-Y. Kim, W.-G. Jung, Variety of nanopatterns on different substrates using PS-bPMMA and their applications, J. Nanosci. Nanotechnol. 12 (2012) 1634-1637 
${ }^{45}$ J. Vieaud, O. Merchiers, M. Rajaoarivelo, M. Warenghem, Y. Borensztein, V. Ponsinet, A. Aradian, Effective Medium Description of Plasmonic Couplings in Disordered Polymer and Gold Nanoparticle Composites, Thin Solid Films 603 (2016) 452-464

${ }^{46}$ B. Maxit, D. Bendejacq, V. Ponsinet, Facile formulation of high density well-ordered nanoparticle-copolymer nanocomposites, Soft Matter 8 (2012) 1317-1320

47 J. J. Chiu, B. J. Kim, E. J. Kramer, D. J. Pine, Control of Nanoparticle Location in Block Copolymers, J. Am. Chem. Soc. 127 (2005) 5036-5037

${ }^{48}$ C. Mendoza, T. Pietsch, J. S. Gutmann, D. Jehnichen, N. Gindy, A. Fahmi, Block Copolymers with Gold Nanoparticles: Correlation between Structural Characteristics and Mechanical Properties, Macromolecules 42 (2009) 1203-1211.

${ }^{49}$ S.-H. Yun, S. M. Yoo, B.-H. Sohn, J. C. Jung, W.-C. Zin, S.-Y. Kwak, T. S. Lee, Electrically Anisotropic Thin Films Consisting of Polymeric and Metallic Nanolayers from Self-Assembled Lamellae of Diblock Copolymers, Langmuir 21 (2005) $3625-3628$

50 B. H. Sohn, B. H. Seo, Fabrication of the multilayered nanostructure of alternating polymers and gold nanoparticles with thin films of self-assembling diblock copolymers, Chem. Mater. 13 (2001) 1752

51 These two additional layers are classically found in BCP thin films simply due to the local conservation of the copolymer composition.

${ }^{52}$ While tuning the film thickness to reach the flat surface cases, it is possible that we obtained in fact a film with a thickness not exactly equal to $n \mathrm{~d}_{\mathrm{o}}$ or $(n+1 / 2) \mathrm{d}_{\mathrm{o}}$. The layered structure of the copolymer then forces the film to present regions of different thicknesses, with different regions satisfying one of the commensurability conditions and the film is therefore terraced, with a quantized surface topography: the step height of the terraces is $d_{o}$, so that the free surface exposes the same block domain on each terrace.

${ }^{53}$ P. F. Green, Wetting and dynamics of structured liquid films, J. Polym. Sci. B: Polym. Phys. 2003, 41, 2219

54 M. Maaloum, D. Chatenay, G. Coulon, Y. Gallot, Edge profile of relief 2D domains at the free surface of smectic copolymer thin films, Phys. Rev. Lett. 68 (1992) 1575

${ }^{55}$ O. Hunderi, K. Johannessen, Effective-medium description of superlattices and the effect on non-locality, Superlatt. Microstruct. 3 (1987) 193-198

56 X.-B. Kang, W. Tan, Z.-G. Wang, Validity of effective medium theory for metal-dielectric lamellar gratings, Opt. Commun. 284 (2011) 4237-4242

${ }^{57}$ P. A. Belov, Y. Hao, Subwavelength imaging at optical frequencies using a transmission device formed by a periodic layered metal-dielectric structure operating in the canalization regime, Phys. Rev. B 73 (2006) 113110

${ }^{58}$ E. Centeno, A. Moreau, Effective properties of superstructured hyperbolic metamaterials: How to beat the diffraction limit at large focal distance, Physical Review B 92 (2015) 045404

59 A. Baron, A. Iazzolino, K. Ehrhardt, J.-B. Salmon, A. Aradian, V. Kravets, A. N. Grigorenko, J. Leng, A. Le Beulze, M. Tréguer-Delapierre, M. A. Duarte, P. Barois, Bulk optical metamaterials assembled via microfluidic evaporation, Opt. Mater. Express 3 (2013) 1792-1797

${ }^{60}$ S. M. Rytov, Electromagnetic Properties of a Finely Stratified Medium Soviet Phys. JETP 2 (1956) 466-475

${ }^{61}$ J. C. M. Garnett, Colours in metal glasses and in metallic films, Phil. Trans. R. Soc. A 203 (1904) 385-420

${ }^{62}$ P. B. Johnson, R. W. Christy, Optical Constants of the Noble Metals, Physical Review B 6 (1972) 4370-4379

${ }^{63}$ T. Repän, A. V. Lavrinenko, S. V. Zhukovsky, Dark-field hyperlens: Super-resolution imaging of weakly scattering objects, Optics Exp. 23 (2015) 25350-25364

${ }^{64}$ S. Ishii, A. V. Kildishev, E. Narimanov, V. M. Shalaev, V. P. Drachev, Sub-wavelength interference pattern from volume plasmon polaritons in a hyperbolic medium, Laser Photonics Rev. 7 (2013) 265-271 